MATEC Web of Conferences 25,03010

(2015)

DOI: $10.1051 /$ matecconf/ 20152503010

(C) Owned by the authors, published by EDP Sciences, 2015

\title{
Research on Transformer Fault Based on Probabilistic Neural Network
}

\author{
Yingshun Li \\ Beijing Institute of Petrochemical Technology, Beijing, China \\ Jingjing $\mathrm{Li}^{*}$ \\ Guangxi University of Technology, Liuzhou, Guangxi, China \\ Junfeng Han \\ Qinzhou University, Qinzhou, Guangxi, China
}

\begin{abstract}
With the development of computer science and technology, and increasingly intelligent industrial production, the application of big data in industry also advances rapidly, and the development of artificial intelligence in the aspect of fault diagnosis is particularly prominent. On the basis of MATLAB platform, this paper constructs a fault diagnosis expert system of artificial intelligence machine based on the probabilistic neural network, and it also carries out a simulation of production process by the use of bionic algorithm. This paper makes a diagnosis of transformer fault by the use of an expert system developed by this paper, and verifies that the probabilistic neural network has a good convergence, fault-tolerant ability and big data handling capability in the fault diagnosis. It is suitable for industrial production, which can provide a reliable mathematical model for the construction of fault diagnosis expert system in the industrial production.
\end{abstract}

Keywords: artificial intelligence; fault diagnosis; expert system; probabilistic neural network

\section{INTRODUCTION}

After the second industrial revolution, the application of a large number of machines promotes the development of human civilization, which is similar to the human medical diagnostic. The most commonly-used diagnosis in industry is the fault diagnosis, and the diagnostic object is the system fault.

There are three stages for the fault model predictions and development course of repair: The first stage is the original stage, just with a simple processing; the second stage is the development stage of testing technology and sensor technology, mainly dealing with signals; the third stage is the stage of intelligent diagnostic techniques for big data processing and forecasting, so that the fault diagnosis achieves considerable development ${ }^{[1]}$. In recent decades, people explore the industrial potential in the bionic algorithm, showing broad application prospects in many research fields.

Currently, the scientific research fields of the bionic algorithm discussed herein include the following aspects:

(1) Expert system of BP neural network.

(2) Expert system of connectionism mechanism. It focuses on the connection with the database and sharing platform, which is suitable for diagnosis in the industrial field.

In the industrial field, the object-oriented programming language and database are widely used for real-time tracking and regulation. For teaching and scientific research, mastering its complex language is not conducive to the students to cultivate their interest This paper constructs a fault diagnosis expert system based on MATLAB. For college students and teachers, it does not require complex and advanced programming, but simply requires calling the function and simple programming. This paper establishes a transformer fault diagnosis expert system based on the practical and efficient principle.

\section{MODELING}

Common transformer fault types are: insulation fault, overheating fault and mechanical failure. Corresponding to the fault, there are specific types. For example, the insulation fault is caused by its aging and moisture; for the discharge fault, there is a need to analyze the kind of discharge fault. The next step is to process according to the fault information detection results so as to predict the development trend and severity, and eliminate the fault when the measures of controlling the fault is proposed ${ }^{[2]}$.

\subsection{BP neural network theory}

BP neural network refers to a kind of multilayer feedforward bionic algorithm. This algorithm has two main features: The first feature is the forward pass of information; the second feature is the reverse transmission of error. There is no interaction between neurons. The change of values has an inherited effect, which is repeatedly recycled until reaching the desired

*Corresponding author: love15040308325@126.com

This is an Open Access article distributed under the terms of the Creative Commons Attribution License 4.0, which permits unrestricted use, distribution, and reproduction in any medium, provided the original work is properly cited. 


\section{MATEC Web of Conferences}

error, and training a matrix that is line with the expected rate of specific gravity ${ }^{[3]}$.

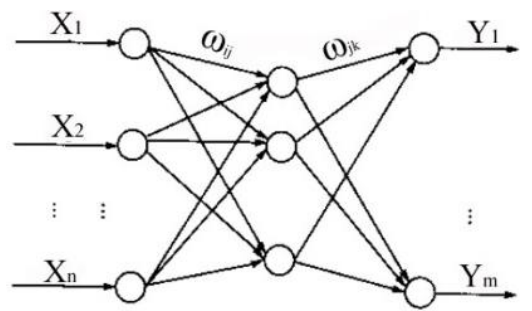

Figure 1. Structure chart of feedforward neural network

In Figure 1, $\mathrm{X}_{1}, X_{2}, \cdots X_{n}$ are input values of forward transmission; $\mathrm{Y}_{1}, Y_{2} \cdots Y_{\mathrm{m}}$ are predicted values of reverse transmission; $\omega_{i j}$ and $\omega_{\mathrm{jk}}$ are specific gravity of the reverse transmission.

As shown in Figure 1, the reverse-transmission neural network is essentially a nonlinear function; the independent variable is an input value of the network; the dependent variable is an output value of the network, thereby building a function relation from the dimension $(\mathrm{n})$ to the dimension $(\mathrm{m})$.

The training network can make data become standard and network more intelligent. The training steps are as follows:

First step: initialize the network. Determine the number of nodes at the typing layer (n), the number of nodes at the hidden layer $(l)$ and the number of nodes at the printing layer $(\mathrm{m})$ according to the typing and printing matrix $(\mathrm{X}, \mathrm{Y})$. Initialize the specific gravity connected between the neurons at the typing layer and the printing layer ( $\omega_{i j}$ and $\omega_{\mathrm{jk}}$ ), the hidden layer range (a), the printing layer range $(b)$ and a given acquisition rate and agitation function.

Second step: output at the hidden layer. Determine the number of nodes at the typing layer (n), the specific gravity connected between the hidden layers $\left(\omega_{i j}\right)$ and the range $(a)$ according to the matrix $(\mathrm{X}, \mathrm{Y})$, so as to calculate the output at the hidden layer $(H)$.

$H_{j}=f\left(\sum_{i=1}^{n} \omega_{i j} x_{i}-a_{j}\right) j=1,2, \cdots, l$

In the Formula (1), $l$ is the number of nodes at the hidden layer; $f$ is an agitation function.

Third step: output at the printing layer. The predicted output $(O)$ of bionic algorithm can be calculated by the output at the hidden layer $(H)$, the specific gravity of connection ( $\left.\omega_{\mathrm{jk}}\right)$ and range $(\mathrm{b})$.

$O_{k}=\sum_{1}^{l} H_{j} \omega_{j k}-b_{k} k=1,2, \cdots, m$

Fourth step: error calculation. The prediction error (e) can be calculated by the predicted printing $O$ and expected printing $Y$.

$e_{k}=Y_{k}-O_{k} k=1,2, \cdots, m$
Fifth step: update of specific gravity. The specific gravity of connection $\left(w_{i j}, w_{j k}\right)$ can be updated according to the prediction error (e).

$w_{i j}=w_{i j}+\eta H_{j}\left(1-H_{j}\right) x(i) \sum_{k=1}^{m} w_{j k} e_{k}$

$j=1,2, \cdots, n j=1,2, \cdots, l$

In the Formula (4), $\eta$ is the learning rate.

Sixth step: update of range. The range $(a, b)$ can be updated to the prediction error (e) in the algorithm.

$$
\mathrm{a}_{\mathrm{j}}=a_{j}+\eta H_{j}\left(1-H_{j}\right) \sum_{k=1}^{m} w_{j k} e_{k}
$$$$
j=1,2, \cdots, l b_{k}=b_{k}+e_{k} k=1,2, \cdots l
$$

Seventh step: determine whether it is finished. If it is not up to standard, return to the second step ${ }^{[4]}$.

\subsection{Probabilistic neural network theory}

The probabilistic neural network was first proposed by Dr. D.F.Specht in 1989. Such neural network is a kind of parallel algorithm based on the probability theory. This algorithm has many advantages, one of which is the outstanding classification capacity and multidimensional processing capability, and the high prediction accuracy.

PNN network is a kind of feedforward bionic algorithm. Its theoretical basis is the minimum risk criteria of Bayes. This algorithm is developed from the radial basis function, which is very suitable for pattern recognition ${ }^{[5]}$.

The algorithm model in this paper consists of four layers, namely the typing layer, the model layer, the weighted layer and the printing layer. The basic structure is shown in Figure 2:

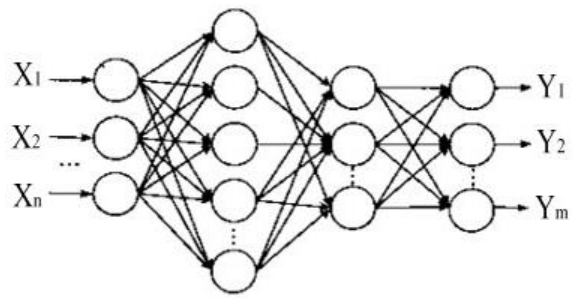

Figure 2. Basic structure chart

The typing value is converted to the eigenvector and passed on to the network at the next layer. The number of dimensions of samples is the number of neurons. A sum of various kinds of training samples is equal to the number of neurons at the model layer. Output of each unit at the model layer is:

$\mathrm{f}(X, W)=\exp \left[\frac{\left(X-W_{i}\right)^{T}\left(\mathrm{X}-\mathrm{W}_{\mathrm{i}}\right)}{2 \delta^{2}}\right]$ 
Where: $W_{i}$ is the specific gravity from the typing layer to the model layer. $\delta$ is a smoothness index.

The weighted layer is to calculate statistical values according to the Formula (6) and obtain PDF. The weighted layer is distributed by category and weighted by category without linking with other units. The greater the probability estimate is, the more output at the weighted output will be. The printing layer carries out further normalization processing.

PNN troubleshooting prediction algorithm is described as follows: assuming that there are two known models, $\theta_{\mathrm{A} \text { and }} \theta_{\mathrm{B}}$. For the fault feature sample $\mathrm{X}=\left(\mathrm{x}_{1}, \mathrm{x}_{2}, \cdots \mathrm{x}_{\mathrm{n}}\right)$ that is to be judged:

$$
\text { If } h_{A} l_{A} f_{A}>h_{\mathrm{B}} l_{\mathrm{B}} f_{B} \text {, then } X \in \theta_{\mathrm{A}} \text {. }
$$$$
\text { If } h_{A} l_{A} f_{A}>h_{\mathrm{B}} l_{\mathrm{B}} f_{B} \text {, then } X \in \theta_{\mathrm{A}} \text {. }
$$

Where: $h_{A}$ and $h_{\mathrm{B}}$ are the prior probability of $\theta_{\mathrm{A}}$ and $\theta_{\mathrm{B}}$ in the fault mode $\left(h_{A}=\frac{N_{A}}{N}, h_{B}=\frac{N_{B}}{N}\right)$; $N_{A}$ and $N_{B}$ are the number of training sample in the accident mode; $N$ is a total number of training samples; $l_{A}$ is a penalty factor of $\theta_{\mathrm{A}} ; l_{B}$ is a penalty factor of $\theta_{\mathrm{B}}$. The so-called penalty factor is to mistakenly divide the sample $\mathrm{X}$ that belongs to $\theta_{\mathrm{B}}$ into $\theta_{\backslash \mathrm{A}} ; f_{A}$ and $f_{B}$ are the probability density function (PDF) in the fault mode $\left(\theta_{\mathrm{A}}\right.$ and $\left.\theta_{\mathrm{B}}\right)$.

Parzen proposed an estimation method in 1962 This method can be used to obtain the probability approximation function. The estimation formula is as follows:

$$
f_{A}(X)=\frac{1}{(2 \pi)^{\frac{\mathrm{P}}{2}} \delta^{P}} \frac{1}{m} \sum \exp \left[-\frac{\left(X-X_{a i}\right)^{T}\left(X-X_{a i}\right)}{2 \delta^{2}}\right]
$$

Where: $X_{a i}$ is the training vector $(i)$ in the fault $\operatorname{mode}\left({ }^{\theta_{\mathrm{A}}}\right)$;

$m$ is the number of training samples in the fault $\operatorname{mode}\left({ }^{\theta_{\mathrm{A}}}\right)$;

$\delta$ is the smoothness factor.

\section{MODEL SOLUTION}

There is a need to pay attention to some problems in the neural network modeling. The selection of eigenvector is a proper reflection of the features of the problem. The diagnosis result often depends on it Whether the feature contains sufficient information to be recognized is very important. The latent fault of the transformer shall be predicted and corrected in troubleshooting timely. The analysis method of gas dissolved in oil can be used to be competent in this work. The input eigenvector in the bionic algorithm is the three contrast value of gas dissolved in oil. The fault type is the output matrix ${ }^{[6]}$.

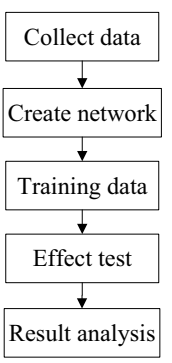

Figure 3. Algorithm step design flow chart

\subsection{Fault diagnosis and simulation}

The data collected is the matrix of $33 \times 4$ dimension. Several former samples (such as 23 samples) are selected as network training samples; several latter samples (such as 8 samples) are selected as validation samples. The input layer of the neural network is the value of three-ratio method; the output is the fault identification. The codes corresponding to the fault type are shown in Table 1:

\begin{tabular}{ll} 
Table 1. Codes corresponding to the fault type & \\
\hline Fault type & Code \\
\hline Partial discharge & 1 \\
Low temperature overheating & 2 \\
Medium temperature overheating & 3 \\
High temperature overheating & 4 \\
Normal & 5 \\
\hline
\end{tabular}

After creation of the bionic algorithm model of the classification forecast of the machine fault, two layers of algorithm network are established on the basis of MATLAB, which are respectively the classification layer (clustering layer) and the competition layer. The creation of PNN network directly uses the function net=newpnn $(\mathrm{P}, \mathrm{T}$,spread $)$ provided by MATLAB. As described above, the input vector and threshold value shall be input in the function after processing ${ }^{[7]}$.

After loading the data, the typing and printing matrix is selected and the desired category is transformed into the vector. This network designed in the algorithm of this paper can be used for training. If the results obtained are qualified, the built-in function can be used for prediction and visualization of the results.

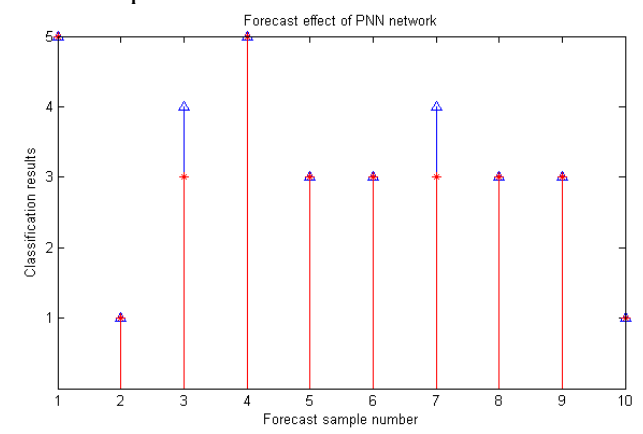

Figure 4. Forecast result 


\section{MATEC Web of Conferences}

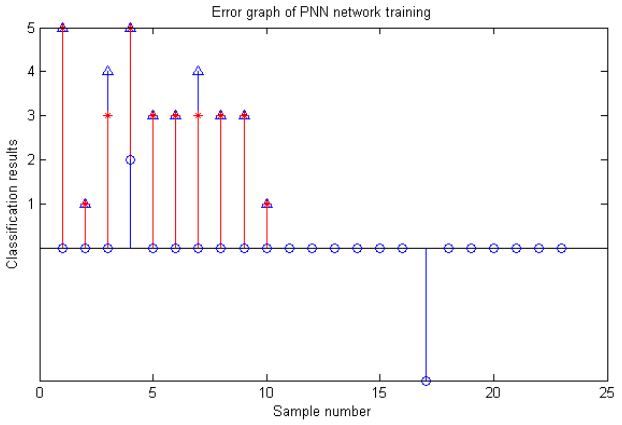

Figure 5. Training residual plot

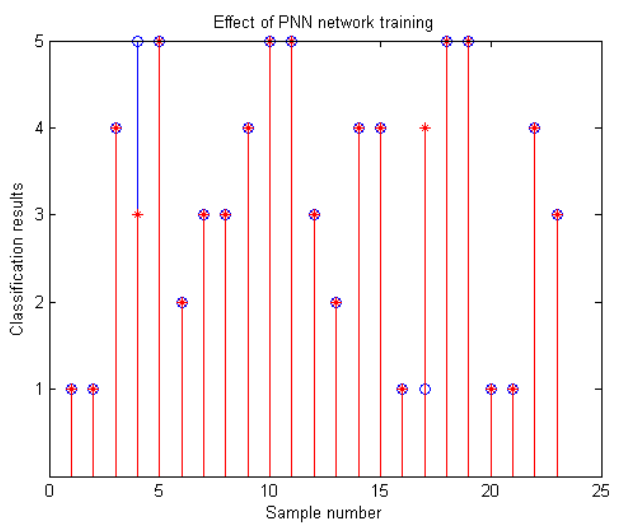

Figure 6. Training result

Table 2. Forecast results of fault diagnosis of probabilistic neural network

\begin{tabular}{lll}
\hline $\begin{array}{l}\text { Number of fore- } \\
\text { cast samples }\end{array}$ & $\begin{array}{l}\text { Forecast classi- } \\
\text { fication results }\end{array}$ & $\begin{array}{l}\text { Actual classifi- } \\
\text { cation results }\end{array}$ \\
\hline 1 & 5 & 5 \\
2 & 1 & 1 \\
3 & 4 & 3 \\
4 & 5 & 5 \\
5 & 3 & 3 \\
6 & 3 & 3 \\
7 & 4 & 3 \\
8 & 3 & 3 \\
9 & 3 & 3 \\
10 & 1 & 1 \\
\hline
\end{tabular}

In the bionic algorithm of this paper, two factors restrict the accuracy. One is the number of training data, and the second is the selection of S values. The research shows that the more sample values and the wid- er types are, the more accurate the forecast results are. However, the lower the spread value is, the more accurate the forecast result is. The simulation in this paper and research in the previous literature shows that for the BP neural network, its convergence rate, solution stability and fault-tolerant capability of sample complementing are to be improved. The algorithm used in this paper has robustness on the above issue. The forecast results are shown in Table 2 .

\section{CONCLUSION}

Through the matrix laboratory simulation, the bionic algorithm in this paper has the following advantages:

(1) The process model is simple and the convergence is good.

(2) The network convergence effect with a good stability is better than the optimal solution of Bayes. It has clear theoretical support, which is convincing and explained reasonably.

(3) The fault-tolerant capability of samples is strong, and the specific gravity is mutually independent. The increase and decrease of the samples are very simple and convenient, and the accuracy of forecast results is high with excellent judgment accuracy.

In conclusion, the diagnostic system of artificial intelligence machine based on the probabilistic neural network has superior performance in terms of speed and fault tolerance.

\section{REFERENCES}

[1] Mao Haoxi. 2007. Research on fault diagnosis of hydraulic system of continuous casting machine. Guangdong University of Technology.

[2] Guo Jianghua, Liang Shuhai, \& Liang Yong. 2003. Fault diagnosis of marine diesel engine based on neural network. Computer Simulation. (08)

[3] Yang Jiangang. 2001. Practical Guide for Artificial Neural Network. Zhejiang University Press.

[4] Yang Hailan, \& Wu Yixiong. 2003. Artificial neural network of principal component analysis of welding quality control. Journal of Shanghai Jiaotong University. (09).

[5] Xiong Jianqiu, \& Li Zuoyong. 2006. Prediction of slope stability of water and electricity by probabilistic neural network. Subgrade engineering. (04)

[6] Wu Mingjiang, Shi Hui, Zhu Xiaohua, \& Xiao Kaiqing. 2005. Current Situation and Prospect of Fault Diagnosis Expert System. Computer Measurement \& Control. (12).

[7] Lexander G Parlos. 1994. An accelerated learning algorithm for multiplayer perceptron networks. IEEE ACM Transactions on Networking. 